\title{
Médiévales
}

Langues, Textes, Histoire

54 | printemps 2008

Frères et sœurs

\section{L'hôpital Santa Maria della Scala : une institution siennoise}

Traduit de l'italien par Didier Boisseuil

The Santa Maria della Scala Hospital : an institution of medieval Siena

\section{Renato Lungarini}

\section{OpenEdition}

\section{Journals}

Édition électronique

URL : https://journals.openedition.org/medievales/5333

DOI : 10.4000/medievales.5333

ISSN : 1777-5892

\section{Éditeur}

Presses universitaires de Vincennes

Édition imprimée

Date de publication : 1 juin 2008

Pagination : $99-111$

ISBN : 978-2-84292-217-7

ISSN : 0751-2708

\section{Référence électronique}

Renato Lungarini, «L'hôpital Santa Maria della Scala : une institution siennoise », Médiévales [En ligne], 54 I printemps 2008, mis en ligne le 10 septembre 2010, consulté le 24 avril 2022. URL : http:// journals.openedition.org/medievales/5333; DOI : https://doi.org/10.4000/medievales.5333

Ce document a été généré automatiquement le 24 avril 2022.

Tous droits réservés 


\section{L'hôpital Santa Maria della Scala : une institution siennoise}

Traduit de l'italien par Didier Boisseuil

The Santa Maria della Scala Hospital : an institution of medieval Siena

Renato Lungarini

1 L'histoire de l'hôpital Santa Maria della Scala, le plus important des hôpitaux siennois médiévaux, placé au pied de la cathédrale, a fait l'objet, ces dernières années, de recherches nombreuses et novatrices. Les travaux pionniers de la seconde moitié du $\mathrm{XIX}^{\mathrm{e}}$ siècle - consacrés essentiellement à l'histoire institutionnelle de l'établissement ${ }^{1}$ - ont été récemment approfondies par un ensemble de chercheurs parfois de jeunes étudiants - à l'occasion des nombreuses manifestations éditoriales (des conférences, des colloques et des expositions) qui depuis les années 1980, accompagnent les transformations de l'hôpital en un centre muséal de première ampleur ${ }^{2}$. Ces enquêtes portent à la fois sur l'histoire de l'établissement entre le XIII et le XIV ${ }^{\text {e }}$ (de ses infrastructures et de leur fonction, essentiellement d'assistance ${ }^{3}$ ) et sur la communauté des fratres Sancte Marie qui joua un rôle social, économique et même politique de premier plan au sein de la cité et de son territoire ${ }^{4}$. L'abondante documentation conservée permet de mettre en lumière le statut singulier du chapitre des frères qui fut, aux XIII ${ }^{\mathrm{e}}$ et $\mathrm{XIV}^{\mathrm{e}}$ siècles, le principal organe délibérant de l'hôpital conçu, à la fois, comme une institution communale et ecclésiastique.

L'hôpital : une institution polyédrique entre établissement ecclésiastique et établissement communal

2 L'hôpital Santa Maria della Scala apparaît pour la première fois mentionnée en mars 1090, dans les documents du chapitre, comme « xenodochio ». Sa création s'insère dans le vaste mouvement de création d'institutions hospitalières nouvelles qui prend essor à la fin du xie siècle, soumises au contrôle de l'évêque ou des chanoines et confiées à des fraternitates laïques. Il répond aux impératifs de l'hospitalitas telle qu'elle fut définie dès le début du Moyen Âge: un service à rendre aux plus nécessiteux et aux malades (attitude qui était demeurée durant l'époque romaine essentiellement une initiative 
individuelle) $)^{5}$. Cette fonction d'assistance connut, par la suite, d'importantes évolutions: elle s'élargit et se différencia en raison de l'importance croissante des fonctions thérapeutiques - gouvernées par des principes médicaux ${ }^{6}$. La plupart d'entre eux devinrent alors des établissements bien plus grands, tant sur le plan institutionnel qu'économique ${ }^{7}$. En moins d'un siècle, l'hôpital siennois devint l'une des institutions les plus importantes de la cité. Il relevait à la fois des mondes laïque et ecclésiastique $^{8}$, recourant, selon les besoins à l'immunité, propre aux établissements religieux ou à la protection de l'autorité communale.

Jusqu'au XII siècle, l'hôpital siennois est étroitement lié à la cathédrale Santa Maria et à la communauté religieuse, formée non seulement de chanoines, mais aussi au groupe de laïcs qui gravitaient autour d'eux et qui avaient choisi de devenir convers'. Prendre l'habit de pénitent et se donner à la communauté hospitalière comme oblat signifiait, certes, rompre avec la vie dans le siècle, mais n'interdisait pas de continuer à entretenir avec ses contemporains, au sein de la cité, des relations personnelles, économiques ou sociales ${ }^{10}$. Ce sont d'ailleurs ces oblats - connus comme les frères de Santa Maria della Scala - qui contribuèrent à former le patrimoine foncier de l'établissement en offrant leur bien, lors de leur entrée dans l'ordre. Les terres qu'ils remettaient s'ajoutaient à celle que l'hôpital pouvait acheter grâce aux aumônes des laïcs pro remedio animarum ou à la dot qu'apportait le recteur nouvellement désigné, qui appartenait aux familles citadines les plus aisées ${ }^{11}$.

Dès la fin du xiII ${ }^{\mathrm{e}}$ siècle, par le biais de ces dons, l'hôpital Santa Maria della Scala devint une puissance économique de première ampleur qui assuma, au cours du siècle suivant, un rôle bancaire important. En effet, l'établissement recevait en dépôts une partie du pécule des pèlerins en route pour Rome et même les espèces des citoyens auxquels il versait des intérêts ${ }^{12}$. L'importance du patrimoine foncier et la place acquise par l'établissement au sein de la société siennoise suscita d'importantes rivalités entre la fraternitas - qui désirait gérer de façon autonome l'hôpital et ses biens -, le clergé qui entendait conserver le contrôle de l'institution - et la commune de Sienne - qui convoitait les richesses et le pouvoir économique et politique de l'établissement.

Dès le début du XIII ${ }^{\mathrm{e}}$ siècle, la commune s'intéressa activement à l'hôpital, en soutenant son développement. D'abord les consuls, puis le podestat s'efforcèrent de défendre les bona et res et iura hospitalis Sancte Marie et donc de préserver le patrimoine détenu par l'institution ${ }^{13}$. Le statut communal de 1262 renferme plusieurs articles qui accordent à Santa Maria della Scala, des privilèges importants en matière fiscale et testamentaire et confient à la Commune le soin de veiller à la bonne administration de l'établissement ${ }^{14}$. Mais, en facilitant l'acquisition de nouveaux biens, en accordant des immunités fiscales et sa protection à tous ceux qui souhaitaient faire un don, la commune empiétait sur l'autonomie de la communauté hospitalière, tout comme le chapitre cathédral qui, dès 1240, avait cherché à contrôler l'activité administrative des frères en leur imposant, pour toute transaction économique, d'obtenir l'accord des chanoines. Le conflit d'intérêt entre les trois protagonistes se prolongea tout au long du XIII siècle, alors que le clergé, pro-impérial, s'opposait à la faction populaire qui accédait au gouvernement de la cité ${ }^{15}$.

6 La plupart des normes édictées concernant Santa Maria della Scala furent reprises dans deux grandes rubriques du statut réalisé par le gouvernement dit des Neuf, en $1287^{16}$. C'est à l'initiative de ce régime que quelques années plus tard la Commune passa de la garde de l'hôpital à un véritable contrôle de l'institution. Ce changement s'effectua à la 
fin du XIII ${ }^{e}$ siècle, alors que la communauté hospitalière paraissait affaiblie et que les autorités urbaines, en raison de l'exiguïté des finances municipales, lorgnaient sur les ressources de l'établissement et celles des citoyens engagés à son service. Désireuse de s'affranchir de la tutelle des clercs, la communauté qui revendiquait son appartenance à la Commune fut contrainte de renoncer à son autonomie en faveur des autorités publiques ${ }^{17}$. Ce choix se révéla judicieux, même si la perte d'indépendance compliqua les relations avec les clercs. L'institution se structura, car c'est à cette époque que furent compilés pour la première fois les statuts de l'Hôpital. À partir du mois de mai 1305 , le chapitre travailla à l'élaboration de ses propres normes, parmi lesquelles figuraient, en premier lieu, l'obligation « d'aimer la Commune de Sienne », de ne pas la voler ou la faire voler ${ }^{18}$. Cette évolution ne fut pas sans conséquence sur le chapitre, composante fondamentale du fonctionnement de l'hôpital.

Le chapitre : organe fondamental du fonctionnement de l'hôpital

7 Le chapitre, en tant qu'organisme délibérant, eut en effet un rôle de premier plan dans la gestion de l'établissement. Il apparaît pour une première fois mentionné dans un acte du 3 mars 1194, conservé dans le Diplomatico Santa Maria della Scala, qui définit ses compétences et établit le transfert de l'administration du patrimoine de l'hôpital des mains des chanoines de la cathédrales à celles des frères ${ }^{19}$. Un document de deux mois antérieurs, autorisait déjà les frères à choisir le recteur ${ }^{20}$ et donc en un temps très bref, le chapitre se vit définir de façon formelle les moyens d'assurer la direction de l'établissement.

8 Les prérogatives assumées par le chapitre de l'hôpital sont comparables à celles assumées par le chapitre de la cathédrale qui gérait une partie des biens dévolus à l'église de la cité - réunis dans la mense canoniale - et choisissait l'évêque depuis la réforme grégorienne ${ }^{21}$.

9 L'activité du chapitre est perceptible grâce à la documentation qu'immédiatement il se mit à produire. Il fut, ainsi, à l'origine d'un ensemble de pièces que nous avons conservées : les actes étendus sur parchemin («pergameni ») et les registres notariés qui permettent de saisir son organisation, sa structure.

10 La base de cette documentation, était formée d'actes notariés qui venaient à instituer un sindacus ou procureur qui avait pour tâche d'agir au nom de l'Hôpital. C'est à l'occasion du sindacatus que s'exprimait la volonté du chapitre. La procuration constituait le moyen juridique par lequel la délibération venait à être appliquée. Les ventes décidées par le chapitre apparaissent ainsi sous la forme: "le chapitre et le recteur décident la nomination d'un frère en qualité de syndic ad vendendum... ». Cette procuration pouvait représenter, au-delà de sa fonction pour l'établissement, un moyen de distribuer des responsabilités de façon régulière, pour gérer les biens meubles et immeubles, les finances de l'hôpital. Ce type de procédé est récurrent pour toutes les activités du chapitre - hormis les oblations - et devient monotone.

11 Un acte de vente, parmi les plus anciens conservés, daté du 15 décembre 1235, établi par le notaire Sachetto di Rustichello in dicto hospitali et réalisé à l'initiative du recteur Cacciaconte, de consensu et voluntate et parabola fratrum meorum, laisse entrevoir une première fois la composition du chapitre qui réunissait alors huit personnes ${ }^{22}$. Une poignée de documents ultérieurs, datant $\mathrm{du}$ milieu $\mathrm{du}$ xIII ${ }^{\mathrm{e}}$ siècle, révèlent que le nombre de frères présents oscillait entre 5 (le 30 juin 1242) et 16 (le 9 août 1238) ${ }^{23}$ et précisent les responsabilités du chapitre: ce dernier assistait le recteur cum expresso consilio et consensu, assurait de l'approvisionnement en grain, nommait les procurateurs 
dépêchés lors des conflits qui opposaient l'hôpital à d'autres établissements ecclésiastiques, résolvait les questions d'héritage et gérait le patrimoine de l'établissement ${ }^{24}$.

12 À partir des années 1260 jusqu'au début du XIV ${ }^{\mathrm{e}}$ siècle, les documents produits par le chapitre sont plus nombreux à être conservés et paraissent plus réguliers : le collège était alors composé en moyenne de 11 ou 12 membres, réunis in dicto hospitali, in refectorio, ut moris est ${ }^{25}$. La mention du réfectoire comme lieu de convocation habituelle apparaît à partir du 5 mai 1285 seulement; auparavant les notaires se limitaient à enregistrer le déroulement des assemblées «dans l'hôpital » ou « là où les frères ont l'habitude de se réunir ».

13 L'existence d'une forme de hiérarchie au sein du chapitre se déduit de la fréquence avec laquelle certaines charges sont confiées à quelques frères. Les mandats de procuration permettent d'individualiser ceux qui avaient le plus de crédit au sein de l'institution et qui assumèrent, souvent durant la même période, la charge de syndic de l'hôpital. Les procurateurs étaient, avec le trésorier, les seuls à s'occuper des aspects financiers de la gestion de l'hôpital et constituaient un groupe restreint au sein du conseil $^{26}$. Rarement des personnes externes à l'établissement se voyaient confier des tâches financières ou judiciaires: les frères expérimentés, quel que soit leur statut social, étaient préférés.

14 La structure sociale du chapitre, malgré le peu de documents disponibles pour le XIII siècle, se déduit du nom des oblats inscrits sur les parchemins. En s'aidant de leur nom, des surnoms toponymiques, de la mention de qualificatifs comme «ser » ou «messer », des métiers qu'ils pratiquaient, il est possible de distinguer, à côté des frères issus de l'aristocratie, tout un ensemble d'oblats d'origine plus modeste (curés, juristes, artisans) ou même populaire.

Les modalités et le déroulement des réunions restent encore obscures. Seuls les formulaires des actes notariés fournissent quelques indications, dans les limites de ce que ce type de document est susceptible de produire. Un mandat de procuration de décembre 1295, concernant la prise de possession de quelques biens situés dans le "castello" de Monteguidi, mentionne pour une première fois le terme de "délibération ${ }^{27}$. Le notaire Ranieri del fu Niccolò notait comment le recteur, en présence et avec l'accord du chapitre, habito diligenti tractato et deliberatione plenaria, avait nommé un certain Buccia di Grazia da Menzano, procureur ${ }^{28}$. Le 5 mai 1298, un autre notaire Orlando del fu Guglielmo, usait de la même formule dans un acte de $v^{v e n t e}{ }^{29}$. L'emploi de l'expression "délibération plénière » n'est pas anodin. En effet, jusqu'à ce que les sessions du chapitre aient été enregistrées dans des cahiers, au milieu du XIV ${ }^{e}$ siècle, et qu'elle révèle la pratique de la discussion (tractato), aucun document ne donne d'indication sur les procédures d'élaboration de la reformatio. Au sein des conseils publics citadins, la décision était établie à l'issue de discussions contradictoires, puis mises aux votes ${ }^{30}$. Dans les actes du «diplomatico» ou les actes notariés provenant de l'hôpital, il n'est pas fait mention d'un vote, ni d'une forme quelconque d'opposition lors des réunions. Bien au contraire, les rédacteurs signalaient que les frères approuvaient la proposition du recteur unanimiter et concorditer, c'est-àdire à l'unanimité et de façon concordante, comme il est d'usage à l'époque. Cette formule se maintint ultérieurement, dans la documentation du XIv ${ }^{\mathrm{e}}$ siècle, avant que le chapitre ne produise des registres, malgré les normes statutaires qui indiquaient clairement que chaque délibération devait être établie à l'aide d'un scrutin. Dans la 
presque totalité des cas, il convient de rappeler que le consentement du collège n'impliquait pas la mise au vote de l'argument débattu, mais la nomination d'une personne chargée d'appliquer les décisions du chapitre. Il s'agissait donc d'un second vote, destiné à être exécutoire, sûrement plus formel et moins discuté que le précédent. Statuts, mémoire et notaire

Les plus anciens statuts conservés de l'hôpital, comme nous l'avons vu précédemment, date de 1305 et règlent la vie de l'ensemble de la fraternitas y compris le chapitre. La rubrique LII accorde aux seuls donnés de la fraternitas la possibilité de gérer l'établissement et attribue au recteur pleine autorité pour convoquer et composer le chapitre $^{31}$. La rubrique suivante confirme le pouvoir du recteur et rappelle que le chapitre ne peut se réunir sans son consentement ${ }^{32}$. Leur rapport est donc réciproque : le chapitre nomme officiellement le recteur, accompagne et coordonne son activité ; ce dernier décide de qui doit l'assister lorsqu'il le souhaite. De ce rapport découle toutes les dispositions statutaires et toutes celles inhérentes à la gestion administrative et patrimoniale de l'établissement: la nomination aux différents offices, les transactions immobilières et financières, les affaires judiciaires. Le recteur peut procéder, toutefois, dans quelques cas particuliers, sans réunir le conseil, mais en rassemblant quelques frères de confiance. L'autorité collégiale apparaît, donc, soumise à celle du recteur, et ne s'impose que lorsque le siège de celui-ci est vacant.

ce même statut de 1305, les normes concernant l'organisation ou le rôle du chapitre restent marginales, hormis les deux rubriques citées précédemment qui se situent à la fin du recueil. Le chapitre apparait seulement mentionné dans quelques passages, comme organe exécutif dont les prérogatives sont établies par la coutume. Le fait qu'au moment de l'établissement du texte, les compétences du collège des frères étaient déjà bien établies, explique peut-être qu'il n'ait pas paru souhaitable de formuler une réglementation de cette organe qui était pourtant à l'initiative du corpus normatif ${ }^{33}$.

Le statut suivant composé en 1318 est bien différent et les dispositions destinées à discipliner le collège sont plus importantes et détaillées. Le chapitre IV ( Du chapitre général ») établissait que le recteur devait convoquer près de l'hôpital un chapitre général de tous les frères "composés tant de ceux qui demeurent dans le "contado " que de ceux qui demeurent à Sienne ", deux fois par an " ou bien une seule fois selon ce qui plaira au recteur $\aleph^{34}$. L'assemblée devait se réunir tous les ans lors des fêtes mariales : à l'Assomption de la Vierge en août et à la Sainte-Marie en mars. Les frères devaient être avisés par un messager ou par une lettre et les absents étaient punis d'une amende de 40 sous pour ceux qui résidaient en dehors de Sienne, d'une peine disciplinaire pour ceux qui vivaient dans le couvent et qui ne présentaient pas d'excuses valables : un jeûne au pain et à l'eau de trois jours ${ }^{35}$. Lors de la réunion, chaque frère était invité à exposer son propre point de vue "pour l'utilité dudit hôpital » et le recteur devait s'engager à respecter ce qui serait décidé par les deux tiers du chapitre ${ }^{36}$. À côté du chapitre général, il existait un chapitre ordinaire chargé de la gestion continuelle et ordinaire de l'établissement. La rubrique $\mathrm{V}$ prévoyait de réunir ce collège au moins deux fois par mois ou plus souvent si le recteur le jugeait

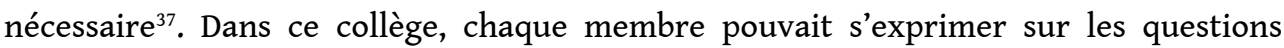
soulevées par le recteur et avait la possibilité de formuler des propositions. La réunion n'avait de valeur que si les deux tiers des participants étaient réunis. Une norme de 
1320 prévoyait d'élever le nombre de présent, au minimum à 15 lorsqu'il fallait traiter des affaires financières, ce qui se produisait une fois par $\mathrm{an}^{38}$.

Les modalités qui permettaient de transformer les délibérations en dispositions exécutives étaient prévues dans la rubrique LXXXII, où sont rappelées les nécessités d'établir "les décisions au sein du chapitre par un scrutin». La norme présentait cependant quelques ambiguïtés : si d'un côté, l'obligation d'effectuer un scrutin « avec urne et boules » (« a bossoli e pallotte ») était prévue «à chaque fois qu'il conviendra de prendre parti au sein du chapitre de l'hôpital sur les choses qui ont de l'importance »; de l'autre, il était laissé au recteur ou à son vicaire le soin de décider « quand le scrutin devait être fait ou non $»^{39}$. Les délibérations contenues dans le «diplomatico» ou le " notarile " portent toujours la mention de décisions établies à "l'unanimité et dans la concorde", sans préciser l'existence d'un scrutin. L'existence formelle d'un scrutin n'apparaît qu'en 1322, dans une délibération établie en vulgaire, placée en ajout du statut de 1318. Ainsi les frères, convoqués par le recteur en chapitre, au son de la cloche, dans le réfectoire de l'hôpital, délibérèrent après avoir « pris parti par le biais d'un scrutin et tous furent d'accord sans qu'aucun ne s'oppose». La forme est légèrement différente, mais le sens est le même. Deux autres assemblées importantes furent établies selon le même procédé : celle concernant l'approbation des statuts de 1305 : firmata et approbata [...] cum consensu Capituli fratrum unanimiter et concorditer, et celle enregistrée dans le protocole du notaire ser Ranieri di Accursio, datant du 28 septembre 1314, établissant l'élection du recteur Giovanni di Chese Tolomei ${ }^{40}$. Dans ce dernier cas, la procédure se fit en deux temps, les frères délibérèrent à l'unanimité de procéder à l'élection du recteur, puis décidèrent de nommer Giovanni Tolomei à cette fonction ${ }^{41}$.

Le statut de 1318 n'apporte pas de modification à celui de 1305 concernant les critères de participations aux réunions, il établit qu' "aucun de ceux qui portent le signe ou l'habit de l'hôpital, et qui n'ont pas été offerts à l'hôpital, en personne et en biens, ne peuvent ni ne doivent venir siéger au chapitre $»^{42}$. La possibilité de participer au chapitre était donc accordée aux seuls oblats ; les médecins, clercs et domestiques ne pouvaient y participer. Si l'on observe les inventaires nominatifs de tous les participants aux réunions et qu'on le confronte aux flux des oblats - établi grâce à ceux enregistrés par les délibérations du chapitre - on constate un renouvellement progressif des membres du chapitre : place était faite aux nouveaux oblats, peu après leur arrivée. Le chapitre paraissait donc plutôt ouvert, mais il n'était accessible qu'aux seuls frères résidant à Sienne, ceux qui détenaient des charges qui les retenaient loin de la cité étaient écartés.

21 Les normes statutaires accordent aux collèges d'amples prérogatives administratives parmi lesquelles les plus importantes demeurent la nomination des titulaires aux différentes charges, la gestion du patrimoine, le contrôle de l'ensemble de la vie de l'hôpital (avec la possibilité d'intervenir à tout moment par des mesures disciplinaires); elles s'étendent jusqu'à la possibilité d'accorder aux membres de la confraternitas la permission de nouer des liens de compérage ou commérage avec d'autres personnes ${ }^{43}$. L'institution pouvait s'immiscer dans les affaires privées voire intimes des frères ou de ceux qui entraient en contact avec eux. Ainsi une délibération du conseil de 1325 oblige le trésorier nouvellement élu, Mino di messer Iacopo, à louer une maison dans le Canto de San Giovanni à un couple marié, moyennant vingt livres de loyer et le versement d'une pension de veuvage pour l'épouse, à condition qu'elle 
demeure chaste ${ }^{44}$. La vie entière des frères, des sœurs comme de toutes les personnes qui travaillaient pour l'hôpital ou bénéficiaient de ces biens était donc gouvernée par un chapitre dont l'autorité considérable n'était pas totalement affranchie de celle du recteur.

Comme pour d'autres ensembles documentaires du monde communal italien, la fin du $\mathrm{XIII}^{\mathrm{e}}$ et le début du $\mathrm{XIV}^{\mathrm{e}}$ siècle marquent un tournant pour l'organisation et la transmission de la mémoire. Les actes jusqu'alors conservés dans des parchemins ou des cartulaires furent de plus en plus fréquemment couchés dans des registres tenus par des notaires. Comme la commune ou l'évêque, l'hôpital commença à user des services de notaires de confiance et ce choix coïncide avec celui de conserver auprès de l'institution les registres notariés.

Les statuts du début du XIV siècle révèlent la volonté de l'hôpital de conserver et de transmettre désormais plus manifestement sa mémoire, "afin que les choses et les affaires dudit hôpital aient plus de poids que jamais ils n'en ont eu ». Les normes de 1305 font ainsi mention d'un frère, «scribe de la chambre » et d'un notaire. Le premier avait pour compétence - ainsi que le précise la rubrique 14 - de compiler les registres financiers ${ }^{45}$. Le frère était tenu d'assister le trésorier pour toutes les écritures "qui seront nécessaires à la chambre ${ }^{46}$ ». Le texte mentionne expressément les registres d'introitus et exitus et ceux établissant le détail des dépenses à rédiger sous forme de registres ordinaires. Il s'agissait que «le recteur et les frères ordonnent et rédigent ces livres qui seront nécessaires pour la clarté des activités des frères de l'hôpital ».

24 Une exigence de clarté était donc devenue nécessaire pour une gestion plus satisfaisante de l'institution. Cela tenait vraisemblablement de l'expansion patrimoniale et des compétences financières de l'établissement. Il apparut souhaitable de constituer un espace autonome et une responsabilité nouvelle aux notaires rattachés directement à l'établissement. La rubrique LI du statut détaille d'ailleurs « les choses [que le notaire de l'hôpital] doit utiliser dans son office ${ }^{47}$ ». La norme révèle combien le notaire n'était pas perçu comme une figure étrangère à l'établissement, appelée pour conférer une valeur juridique aux actes de quelque nature qu'ils soient, mais qu'il s'agissait d'un agent intégré à l'institution et qui lui était soumis. Après avoir juré d'agir et d'opérer loyalement, en promettant «de faire tout ce qu'il devra pour l'hôpital, le recteur, les frères, les sœurs et les familiers ", il devait accepter de tenir des livres "d'imbreviature » exclusivement pour les actes de l'hôpital, de rédiger le plus vite possible « les demandes qui lui seront faites, les promesses, les pactes et les obligations et tout ce qu'il revient au notaire d'écrire ». Les documents ne devaient plus être conservés auprès du notaire, mais auprès de l'institution, dans le bureau du trésorier, pièce de laquelle ils ne pouvaient sortir " même si le notaire devait quitter l'hôpital ${ }^{48}$ ". Ainsi, les documents relatifs à Santa Maria della Scala étaient désormais considérés comme relevant de l'hôpital et non plus du rédacteur. Le notaire était, par ailleurs, tenu au secret professionnel, lorsque le recteur ou le chapitre le lui notifiait expressément, alors qu'il pouvait agir à sa guise le reste du temps. Il lui était interdit d'établir des actes qui auraient été contraires aux intérêts de l'hôpital et était obligé de signaler au recteur tous les passages qui pouvaient apparaître comme contraire à l'honneur de Santa Maria ou de la fraternitas ${ }^{49}$.

Le statut de 1318 manifeste un changement important: la rubrique 128 précise qu'il devrait y avoir un second notaire pour faire face aux besoins croissants de productions 
d'actes. Il devait lui aussi résider dans l'établissement, s'occuper des questions de l'hôpital dans le "contado » et accompagner et aider, lors de leur déplacement, les frères qui agissaient pour l'établissement ${ }^{50}$.

La charge de notaire officiel de l'établissement pouvait être assumée par les frères euxmêmes. Le caractère exclusif du lien qui unissait le notaire à l'hôpital ressemble au rôle qu'assumaient à la même époque les notaires pour les différents offices citadins ${ }^{51}$. Il y avait désormais un notaire de l'hôpital (et non plus au service de l'hôpital), tout au moins pour la durée de son engagement au service l'institution. Le fonction de ce notaire s'accrût au cours du XIV siècle, car c'est à lui que revint la charge d'établir toute la documentation émanant de l'hôpital, non seulement les actes nécessitant une reconnaissance authentique, mais aussi les règlements intérieurs, les statuts, les écritures judiciaires, les comptes-rendus de délibération, toutes les pièces nécessitant la fides publica plus que la compétence des différents scriptores.

En ce sens, cette institution reflète bien l'une des caractéristiques de la "civiltà comunale », c'est-à-dire, selon les propos de Gian Giacomo Fissore, «l'expansion du rôle de la documentation écrite, et du rôle du notaire dont l'activité investit tous les moments de la vie, de façon presque maniaque $! »^{52}$.

\section{NOTES}

1. L. BANCHI, Statuti volgari de lo Spedale di Santa Maria Vergine di Siena, scritti l'anno MCCCV ed ora per la prima volta pubblicati, Sienne, 1864 ; ID., Statuto dell'Ospedale di Siena, in Statuti senesi scritti in volgare ne' secoli XIII ${ }^{e} \mathrm{XIV}^{e}$ pubblicati secondo i testi del Regio Archivio di Stato in Siena, vol. III, Bologne, 1877. Le statut a été réédité récemment par M. PELLEGRINI, La comunità ospedaliera di Santa Maria della Scala e il suo più antico statuto: Siena, 1305, préf. A. Bartoli Langeli, Pise, 2005 ; L. BIANCHI, I Rettori de lo Spedale di Santa Maria della Scala di Siena, Bologna, 1877. Toujours au XIX ${ }^{\mathrm{e}}$ siècle, D. BARDUZZI, Del governo dell'ospedale di Siena dalle origini alla caduta della Repubblica, Sienne, 1895 ; G. SANESI, L'origine dello spedale di Siena e il suo più antico statuto, Sienne, 1898.

2. Concernant l'histoire architecturale et archéologique de l'édifice, $c f$. D. GALLAVOTTI CAVALlERO, A. BROGI, Lo Spedale di Siena. Fatti urbanistici e architettonici del Santa Maria della Scala, Florence, 1987 ; Santa Maria della Scala: archeologia ed edilizia sulla piazza dello Spedale, éd. E. Boldrini et R. Parenti, Florence, 1991 ; Siena : lo Spedale di Santa Maria della Scala, édition P. Panza et G. Tucci, Florence, 1996.

3. La società del bisogno. Povertà e assistenza nella Toscana medievale, Florence, 1989 ; G. PICCINNI, « L'Ospedale di Santa Maria della Scala di Siena. Note sull'origine dell'assistenza sanitaria in Toscana (XIV-XV secolo) ", Città e servizi sociali nell'Italia dei secoli XII-XV, Atti del Convegno Internazionale di Studio di Pistoia (9-12 ottobre 1987), Bologne, 1990, p. 297-324 ; ID., « Medici e speziali nell'ospedale di Santa Maria della Scala tra Duecento e Cinquecento ", dans Congresso nazionale dell'Accademia Italiana di Storia della Farmacia, Sienne, 1993, p. 47-54 ; ID., « Tra scienza e arti : lo studio di Siena e 
l'insegnamento della medicina (secoli XIII-XVI) ", dans L'Università di Siena. 750 anni di storia, Milan, 1991, p. 145-158.

4. R. LUGARINI, «Espansione di un ospedale nel Trecento. Spazi, ambienti, cantiere : $\mathrm{i}$ documenti », dans Fonti e materiali per la storia di Siena e del territorio. Un laboratorio di ricerca l'ospedale di Santa Maria della Scala; B. SORDINI, « Per un'enciclopedia multimediale della vita materiale di un ospedale medievale », dans Fonti e materiali, op. cit.; A. CARNIANI, "Censimento dei membri della comunità ospedaliera (XIII-XIV secolo) », dans Fonti e materiali, op. cit.

5. Cf. A. VAUCHEZ, Ordini mendicanti e società italiana. XII-XIV secolo, Milan 1990, p. 221-230. Le document le plus ancien mentionnant l'hôpital est édité dans A. GHIGNOLI, Carte dell'Archivio di Stato di Siena. Opera metropolitana (1000-1200), intr. S. P. P. Scalfati, Sienne, 1994, n. 31, p. 87-90.

6. Durant la première phase du développement hospitalier, la médicalisation était l'aspect le moins important et devint prédominant par la suite, $c f$. G. ALBINI, Città e ospedali nella Lombardia medievale, Bologne, 1993, p. 9.

7. M. MOLLAT, «Complexité et ambiguïté des institutions hospitalières : les statuts d'hôpitaux (les modèles, leur diffusion et leur filiation) », dans Timore e carità. I poveri nell'Italia moderna, Crémone, 1982, p. 3-12.

8. Cf. M. PELLEGRINI, « Le carte in causa ", dans Fonti e materiali, op. cit.

9. M. PELLEGRINI, «L'ospedale e il Comune, immagini di una relazione privilegiata », dans Arte e assistenza a Siena. Le copertine dipinte dell'Ospedale di Santa Maria della Scala, catalogo della mostra (Siena, 7 marzo - 31 agosto 2003), éditions G. Piccinni, C. Zarrilli, Pise, 2003, p. 29-45, p. 30. Cf. c. D. FONSECA, « I conversi nelle comunità canonicali, in I laici e la 'societas' cristiana dei secoli XI e XII », Atti della III settimana internazionale di studio (Mendola, 21-17 agosto 1965), Milan, 1968, p. 262-305.

10. M. PELLEGRINI, Chiesa e città. Uomini, comunità e istituzioni nella società senese del XII e XIII secolo, Rome, 2004, p. 206.

11. Sur les différentes formes d'expérience religieuse à l'intérieur même de l'hôpital, $c f$. O. REDON, « Autour de l'Hôpital Santa Maria della Scala à Sienne au XIII siècle », Ricerche Storiche, XV, 1985, p. 17-34.

12. G. PICCINNI, «L'ospedale e il mondo del denaro » dans Arte e assistenza a Siena, op. cit., p. 17-27, p. 20.

13. Pour la documentation du Duecento, $c f$. R. LUGARINI, « Espansione di un ospedale nel ‘300, ambienti, aree, cantere del Santa Maria della Scala : i documenti », dans Fonti e materali, op. cit., I.21.

14. Il Constituto del Comune di Siena dell'anno 1262, éd. L. Zdekauer, Milan, 1897, I.31, I.34.

15. M. PELLEGRINI, Chiesa e città, op. cit., p. 204-205.

16. Archivio di Stato di Siena (désormais ASs), Statuti di Siena, 3, fol. 10-13, rubr. I.7 e I.8. Le gouvernement dit des "Neuf", composé principalement de riches marchands et banquiers guelfes, dirigea la cité entre 1287 et 1355, après l'éviction du pouvoir des gibelins.

17. Ass, Diplomatico Ospedale Santa Maria della Scala (désormais Diplomatico Ospedale), 1297, février 19.

18. L. BANCHI, Statuti volgari, op. cit., p. 2-4.

19. Ass, Diplomatico Ospedale, 3 mars 1194.

20. Ass, Diplomatico Ospedale, 17 juin 1194. 
21. G. G. MERLO, « Proprietà ecclesiastiche e potenza delle chiese vescovili », dans Storia dell'Italia religiosa, I, Rome-Bari, 1993, p. 293-309, p. 297.

22. Ass, Diplomatico Ospedale, 1235,15 décembre.

23. Ibid., sub data.

24. Cf. Ass, Diplomatico Ospedale, 9 août $1238 ; 8$ janvier $1239 ; 4$ décembre $1241 ; 16$ mars $1241 ; 30$ juin $1242 ; 4$ août 1249 .

25. "Confratres et oblati dicti hospitalis cohadunati in dicto hospitali in refectorio ut moris est ", ASS, Diplomatico Ospedale, 8 mai 1285.

26. Sur les compétences du trésorier, cf. le chapitre XII du statut de 1318 ("De l'officio del camarlingo"), L. BANCHI, Statuti senesi, op. cit., p. 22-23.

27. Ass, Diplomatico Ospedale, 12 décembre 1295.

28. Ibid., « Rector, in presentia et de voluntate et licentia, consensu et auctoritate infrascriptorum fratrum [...] una cum dicto rectore et eius auctoritate, cohadunati in dicto hospitali in loco ubi capitulum fratum dicti hospitalis fieri consuevit, habito diligenti tractato et deliberatione plenaria que sunt capitulum dicti hospitalis nomine et vice dicti hospitalis et capituli et conventus, fecerunt "

29. ASs, Diplomatico Ospedale, 5 mai 1298.

30. P. CAMmAROSANO, Italia medievale, Rome, 1991, p. 164.

31. «Anco stanziamo e stanziando voleno, che nessuno lo quale porti el segno e lo abito del detto Spedale con la persona e con tutti li soi beni, possa e debbia avere alcuno offizio nel detto Spedale, né debbia venire a Capitolo, o vero stare con lo Rettore e frati del detto Spedale quando se farà, s'esso non fusse chiamato a Capitolo per lo Rettore del detto Spedale, o vero fusse dato a lui offizio per esso Rettore. E lo Rettore del detto Spedale possa et a lui sia licito di chiamare e di fare chiamare a Capitolo colui e coloro li quali vorrà, e tante volte quante a lui piacerà, e a lui parrà che se convenga per utilità del detto spedale, secondo che suta è usanza di qua dietro ", Statuti volgari, op. cit. p. 71-72.

32. Ibid., p. 72 : « Anco, che li frati del detto Spedale non possano fare Capitolo né alcuno ordinamento o vero trattato per li fatti e de li fatti del detto Spedale o vero de li frati senza licenzia e volontà de lo Rettore del detto Spedale. »

33. L. BANCHI, Statuti volgari, op. cit., p. 86-87.

34. L. BANCHI, Statuti Senesi, op. cit., p. 15-16.

35. Ibid.

36. «E che in ciascuno de li Capitoli dica e propona qualunche de quelli frati vorrà alcuna cosa dire o vero proponere per utilità del detto Ospitale », ibid.

37. « Anco statuimo et ordinamo, che ' 1 rectore sia tenuto e degga due volte in ciascuno mese, de quindici in quindici dì, fare Capitolo de' frati del detto Ospitale, et in quel Capitolo dire e proponere quelle cose le quali saranno da dire e proponere ad utilità e commodità del detto Ospitale [...]. » Ibid., p. 16-17.

38. «E tutto quello che per esso rectore e Capitolo, o vero due parti di quello, sarà fermato, così sia tenuto el decto rectore di mandare o vero di far mandare ad execuzione [...]. E che in quel Capitolo ciascun frate d'esso Ospitale possa consilliare e dire quelle cose le quali esso frate, el quale sarà presente al detto Capitolo, vedarà e cognosciarà convenire e bisognare per li facti et utilità del detto Ospitale ", Ibid.

39. L. BANCHI, Statuti Senesi, op. cit., p. 79.

40. L. BANCHI, Statuti volgari, op. cit., p. 86-87.

41. ASs, Ospedale, $86 \mathrm{a}, \mathrm{f}^{\circ} 1 \mathrm{r}^{\circ}-3 \mathrm{r}^{\circ}$. 
42. L. BANCHI, Statuti volgari, op. cit., p. 77.

43. L. BANCHI, Statuti senesi, op. cit., p. 122.

44. ASs, Ospedale, $87 \mathrm{a}, \mathrm{f}^{\circ} 87 \mathrm{v}^{\circ}-88 \mathrm{r}^{\circ}$.

45. L. BANCHI, Statuti volgari, op. cit., p. 24 : «Che sia uno de li frati scrittore de la camera del detto spedale, el quale scriva l'entrate e l'essite secondo che farà el camarlingo medesimo ».

46. Ibid.

47. « Come colui el quale sarà notaio del Spedale, debbia giurare ; e che cose debbia usare in suo offizio ». Ibid., p. 69-70.

48. Ibid.

49. «E che non farà né farà fare alcuno instrumento o vero instrumenti in preiudicio $\mathrm{e}$ danno del detto Spedale: e chesto impromettarà di servare a buona fè senza frode. $\mathrm{E}$ s'esso sentisse alcuna cosa la quale ritornasse in danno, o vero incontra lo onore del Rettore o vero de li frati o vero de le donne del detto Spedale; sia tenuto di dire e di manifestare chella cosa al Rettore al più tosto che potrà a bona fè senza frode ", ibid. 50. « e così grande peso non possa essere sofferto per uno notaio, ad ciò che l'Ospitale imperciò non riceva danno, statuimo et ordinamo, che oltra el notaio el quale è ora nel detto Ospitale, degga el detto Ospitale averne uno altro notari, el quale abiti nel detto Ospitale, e facia le questioni de l'Ospitale nelle corti, quando serà bisogno, et acompagni li frati per la città di Siena, et altro du' fusse bisogno per lo detto Ospitale ; e tutte altre cose faccia, le quali incontrassero da fare per li facti del detto Ospitale, secondo e quando inde serà richiesto ", L. BANCHI, Statuti senesi, op. cit., p. 118.

51. BANTI, Studi di storia e diplomatica comunale, Rome, 1983, p. 48-56 ; G. CENCETTI, « Il notaio medievale italiano ", dans Atti della società ligure di Storia Patria, IV (1964), p. XXI. Sur le notariat siennois comme corporation, $c f$. G. CATONI, Statuti senesi dell'Arte dei giudici e dei notai del secolo XIV, Rome 1972 ; ID., « Il collegio notarile di Siena », dans Il notariato nella civiltà toscana, Rome, 1975, p. 339-363 et U. MORANDI, « Il notaio all'origine del Comune medievale senese ", dans Il notariato nella civiltà toscana, op. cit., p. 313 et sq. 52. G. G. FISSORE, « Alle origini del documento comunale : i rapporti fra i notai e l'istituzione ", in Civiltà comunale, op. cit., p. 99-128, p. 101. La difficulté a été soulevée au début du XIX ${ }^{\mathrm{e}}$ siècle par P. TORELLI, Studi e ricerche di diplomatica comunale, I, dans Atti e Memorie della R. Accademia Virgiliana di Mantova, IV (1911), p. 11 et sq. Cf. aussi G. G. FISSORE, « La diplomatica del documento comunale fra notariato e cancelleria. Gli atti del Comune di Asti e la loro collocazione nel quadro dei rapporti fra notai e potere ", dans Studi Medievali, 3, XIX, 1978, p. 211.

\section{RÉSUMÉS}

L'hôpital Santa Maria della Scala naît comme un "xenodochio ", attesté pour la première fois à la fin $\mathrm{du} \mathrm{XI}^{\mathrm{e}}$ siècle, pour devenir par la suite l'une des institutions les plus importantes de l'Italie médiévale. Il fait actuellement l'objet de nombreuses études grâce à des archives presque intégralement conservées et eut un rôle considérable dans l'histoire religieuse, sociale et économique de la Commune de Sienne. Ce présent article permet de mettre en lumière les formes 
de production et de transmission des documents écrits et de reconstruire les structures institutionnelles de l'établissement, ainsi que ses fonctions, à travers l'analyse de sont principal organe de délibération : le chapitre.

The Santa Maria della Scala Hospital : an institution of medieval Siena. The Santa Maria della Scala hospital was born as "xenodochio" whose first traces date back at the and of the eleventh century, to later become one of the most important hospital institution of medieval Italy. The hospital as been object of many researches due to an unaltered archive and had always a relevant role in the religious, social and economic history of the "Comune" of Siena. Today we can rebuild the articulate institutional structure of the hospital with its functions, thanks to the good care towards all forms of production and transmission of the written sources, administrated by the main managing body : the Chapter.

INDEX

Keywords : archives, chapter, hospital, Italy, Siena

Mots-clés : chapitre, hôpital, Italie, Sienne

\section{AUTEUR}

RENATO LUNGARINI

Università degli Studi, Sienne 